\title{
A Systemic Functional Study of the English Nominal Group with $a$ number of
}

\author{
Manliang $\mathrm{Li}^{1}$ \\ ${ }^{1}$ School of Foreign Languages, Inner Mongolia University, Hohhot, China \\ Correspondence: Manliang Li, School of Foreign Languages, Inner Mongolia University, Hohhot 010021, Inner \\ Mongolia Autonomous Region, China. Tel: 86-0471-499-6235. E-mail: limanliang95@sina.com.cn
}

\author{
Received: August 17, 2014 Accepted: September 3, 2014 Online Published: October 1, 2014 \\ doi:10.5539/ijel.v4n5p117 URL: http://dx.doi.org/10.5539/ijel.v4n5p117
}

\begin{abstract}
The literature to date indicates that a number of is a frequently-used expression in the English nominal group. There are various debates over its syntactic function in the nominal group and its internal structure. Namely, the traditional approach, the transformational approach, and systemic functional approach have different description and analysis of this expression. This paper aims to give a delicate analysis of its internal functional structure, from a systemic functional perspective. More specifically, the theoretical basis is the Cardiff Grammar, one register of systemic functional linguistics. The results show that a number of as a whole is a numerical expression in the English nominal group. Its internal structure can be investigated in a novel way.
\end{abstract}

Keywords: English nominal group, a number of, systemic functional linguistics

\section{Introduction}

This paper aims to explore the English nominal group with the numerical expression a number of. The analytical principle is to examine how the meaning is realized by various forms. We begin our analysis by summarizing the previous studies of the nominal group of this kind, including the traditional approach in Section 2 , the transformational approach in section 3, and the systemic functional approach in section 4. In Section 4, we mainly deal with the Sydney Grammar analysis, the Cardiff Grammar analysis and also the analysis by Prakasam. Then, Section 5 is the discussion in which we will go on to elaborate our contentions in the framework of Systemic Functional Linguistics (SFL hereafter) surely but from a perspective of little difference. More specifically, the theoretical framework is the Cardiff Grammar, one dialect of SFL. Last section is the summary and conclusion which summarizes the whole paper and comes to the conclusion.

\section{Traditional Analysis}

In this paper, we shall use a number of as a representative example for the constructions alike. The section that follows will focus on the delicate analysis of the nominal group in (1), and give a comparative analysis of the two nominal groups in (1) and (2) that are similar in the formal structure but dissimilar in the functional structure.

\section{(1) A large number of those books \\ (2) The large number of those books}

At the first glance, these two nominal groups share the equivalent structure in that they are distinguished from each other only by the choice of two different words at the same location, the definite determiner the and the indefinite determiner $a$. In the traditional analysis, number is treated as the Head in both examples, and the elements before and after the Head are the Modifier and the Qualifier, respectively. Huddleston \& Pullum (2002) argue that the predicate verb in a clause does not have to agree in number to the Head of the matrix nominal group that functions as the Subject, as can be seen from the following example (3):

(3) A number of boys were absent (Huddleston \& Pullum, 2002, p. 56)

In this clause, the Subject is the nominal group a number of boys, whose Head is number, which is in singular form, but the predicate verb were is in plural form. This means that the predicate verb were does not accord with the Head number but with another element boys within the nominal group.

Huddleston \& Pullum (2002) give a detailed analysis of the nominal group with a number of as is illustrated in 
Figure 1 below. The nominal group consists of a Determiner and a Head, and the Head is realized by a Nominal, which is the element left in the nominal group when the Determiner is removed. In this analysis, the item number is not the Head of the matrix nominal group but the Head of the Nominal. In addition, the item of is regarded as a common preposition and is dependent upon the prepositional phrase. This analysis does not make any distinction between the constructions in (1) and (2). In other words, the large number of those books and a large number of those books have absolutely the same functional structure. In the semantic perspective, this analysis does not disclose the actual internal logical relationship between the elements.

Huddleston \& Pullum (2002) employ the following examples (4) and (5) to argue that the item of is a constituent with the oblique protesters, not with number. In (4), a number is the abbreviated form of a number of students, and it is of students that is omitted; in (5), of whom a number is equivalent to a number of whom. Thus, the item of in both cases is attached to the oblique rather than to number.

(4) Most students like continuous assessment but [a number _ prefer the old examination system].

(5) We called a meeting of the first-year students, [of whom a number _ had complained about the assessment system].

Quirk et al. (1985) also discuss the nominal group with of-phrase by analyzing the following two examples in (6) and (7), and the analysis reaches a different viewpoint from that by Huddleston \& Pullum (2002).

(6) Of ten reviewers that there were, only a few praised his play.

(7) There were ten reviewers, but only a few praised his play.

(Quirk et al., 1985, p. 1288)

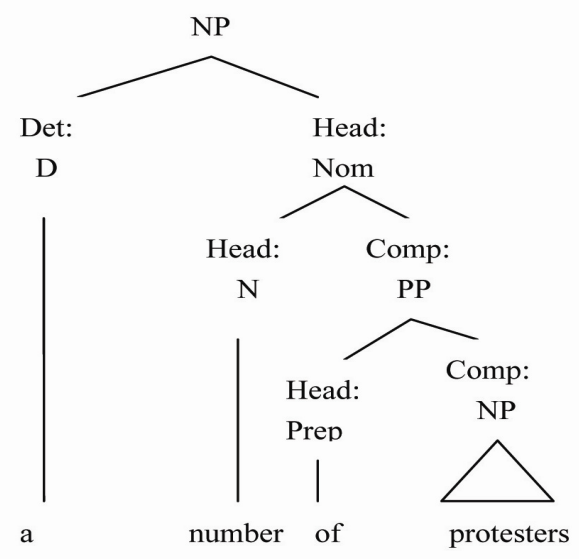

Figure 1. The Traditional analysis of the nominal group with a number of

(Huddleston \& Pullum, 2002, p. 351)

Quirk et al. (1985, p. 1288) treat of ten reviewers as the adverbial of the clause rather than the misplaced Qualifier of the nominal group. The reason is that while (5) is acceptable only a few of ten reviewers is not. In this sense, it is not appropriate to consider of as a common preposition in these cases.

There is another way of looking at the structure of the nominal group like the ones just presented. Namely, the expression a number of as a complete construction is treated as the Quantifier, and this construction consists of a nominal group a number and of (Note 1). Alexander (1991, p. 173) gives examples to show the distinction between a lot of students and a lot of the students and argues that the former nominal group indicates the non-specific reference while the latter denotes the specific reference. In other words, the nominal group a large number of books has a general reference, and in contrast a large number of those books has a specific reference.

Huddleston (1984) puts forward several possible ways of thinking about the structure of the nominal group containing a lot of. Obviously, both a lot of and a number of have the equivalent formal and functional structure. While the analysis of a number of books varies much as a result of the different identifications of the Head of the matrix nominal group, the approach of analyzing the group the number of those books is relatively unanimous. In this nominal group, it is widely acknowledged that number is the Head, and of-phrase is the Postmodifier. 
To sum up, there are two general ways of identifying the Head of the matrix nominal group a number of those books, one way treating number and the other way treating books as the Head. As a result, there are correspondingly two different analyses of the same nominal group. This is the traditional approach to the depiction of the English nominal group with a number of.

\section{Transformational Analysis}

The transformational analysis of the two nominal groups in (1) and (2) must be identical, as is shown in Figure 2. As with the analysis of other types of nominal groups, this approach gives each element in the group a label, and these are labels of word class and group class. Although the internal relationship is clearly shown in the figure, the function of the elements is not clarified and it is still necessary to reconsider the internal structure of the whole nominal group in this approach.

Yet, the transformational analysis resembles the traditional approach. Specifically, although this approach does not use the term Head, Premodifier, Postmodifier to label the element, we can see that $\mathrm{N}$ is treated as the Head, Det and A as the Premodifier, and PP as the Postmodifier.

The transformational analysis indicates the immediate constituency within the matrix nominal group, and this is similar to the embedding or the dependency relationship between the elements. In this example proper, PP is the immediate constituent of the matrix nominal group, and P is the immediate constituent of PP. In our terms, PP is embedded in or dependent on the matrix nominal group and $\mathrm{P}$ is embedded in or dependent on PP.

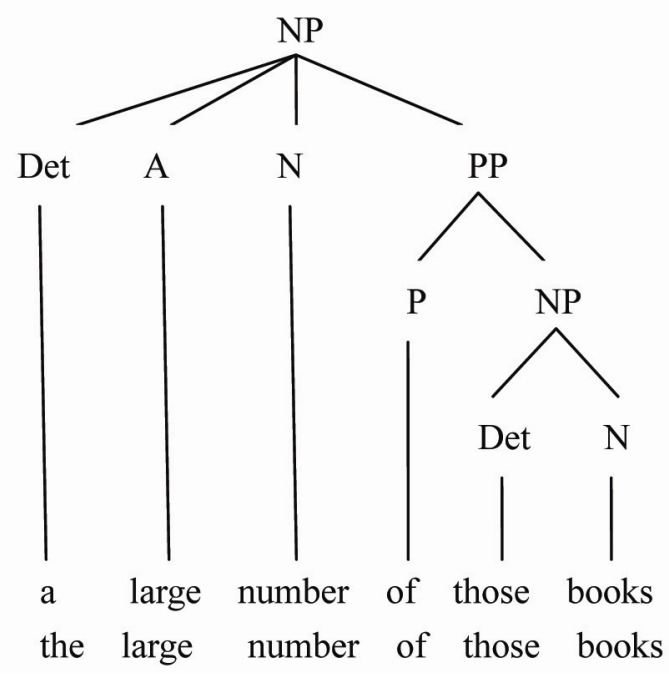

Figure 2. The Transformational analysis of the nominal group with a number of ( $\mathrm{N}=$ Noun; A=Adjective; $\mathrm{P}=$ Preposition; Det=Determiner; NP=Noun Phrase; $\mathrm{PP}=$ Prepositional Phrase)

\section{Systemic Functional Analyses}

The systemic functional approach has its own specific understanding of the two nominal groups in (1) and (2), and the depiction of the nominal group in (2) seems to be similar to the traditional approach and the transformational approach presented in the above sections. The main debate occurs only in the description of the nominal group in (1).

\subsection{The Sydney Grammar Analysis}

Halliday (2000) treats a pack of in the nominal group a pack of cards as the Numerative and cards as the Thing in the experiential structure. From a logical perspective, however, the Head is pack, and $a$ and of cards are respectively the Premodifier and the Postmodifier. Halliday (2000) regards a number of as an item functioning as the Numerative in the nominal group. The quantifying Numerative expresses either an exact or an inexact number. Expressions such as a number of, a lot of, a great deal of specify inexact quantity, while cardinal numerals denote exact quantity. Thus, we can formulate the SFL description of the nominal group in (1) as is shown in Table 1 below. 
Table 1. The SFL analysis of the nominal group with a number of

\begin{tabular}{|c|c|c|c|c|c|c|}
\hline nominal group & $a$ & large & number & $o f$ & those & books \\
\hline experiential structure & \multicolumn{4}{|c|}{ Numerative } & Deictic & Thing \\
\hline \multirow{2}{*}{ logical structure } & \multicolumn{2}{|c|}{ Premodifier } & Head & & Postmodifier & \\
\hline & $\gamma$ & $\beta$ & $\alpha$ & & $\beta$ & \\
\hline lexicogrammar & $\operatorname{det}$ & adj & $\mathrm{n}$ & $\mathrm{p}$ & det & $\mathrm{n}$ \\
\hline
\end{tabular}

Bloor \& Bloor (2001) and Thompson (2000) hold basically the same view as Halliday (2000). The general impression is that they do not provide the delicate analysis of the structure of a number of in the experiential perspective. In addition, it seems that Halliday (2000) does not insist that the structure of five of those books and a number of those books is identical, for he does not include of as a part of the Numerative in five of those books. Moreover, he does not mention the function of of in this kind of structure.

As such, the logical structure of the nominal group in (1) deserves reconsideration. The functional structure of $a$ number of those books is distinguished from that of the number of those books and five of those books. In other words, this nominal group has its own special structure, which will be the focus of the present paper. The section right below will look at how the Cardiff Grammar depicts the nominal group of this type, as it puts forward the delicate analysis of the function of every item within the matrix nominal group.

\subsection{The Cardiff Grammar Analysis}

The Cardiff Grammar (Fawcett, 2000, 2006, 2007, 2008) approach to the description of the nominal group with the quantifying expression a number of deserves attention. It was pointed out in the above section that Halliday (2000) does not conduct a further analysis of the functional structure of a number of by delicacy. The Cardiff approach carries out a detailed exposition in which the function of each element is set forth. Figure 3 below shows the Cardiff Grammar analysis of the nominal group in (1).

Obviously, the Cardiff Grammar assumes that the nominal group a large number of those books has the same functional structure as five of those books. This means the quantifying selection exists in the construction of $a$ large number of those books. In this analysis, a large number is regarded as the quantifying determiner, of as the selector, those as the deictic determiner, and books as the Head. Besides, a large number is another nominal group that fills the element qd (quantifying determiner). This nominal group is composed of three elements, the quantifying determiner $a$, the modifier large, and the Head number.

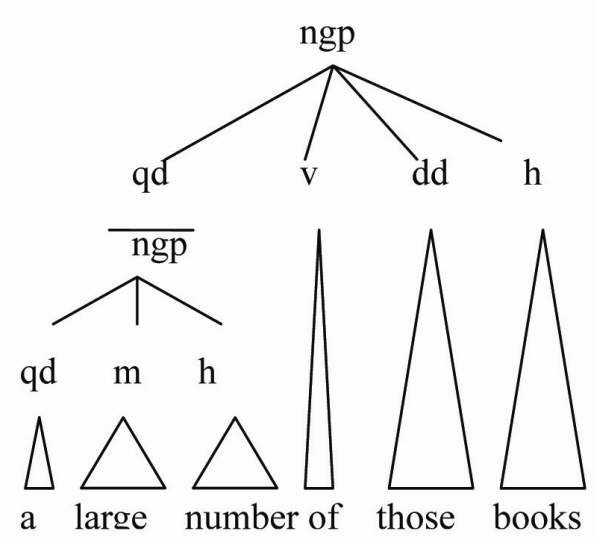

Figure 3. The structure of a nominal group with 'selection' (Fawcett, 2006, p. 194)

The Cardiff approach does not include of as an element of the construction a number of, but an element of the matrix nominal group. In other words, of is the immediate constituent of the nominal group rather than that of $a$ number of. Namely, of is embedded in the matrix nominal group rather than in the numerical expression. In this sense, it is a large number instead of a large number of that functions as the quantifying determiner. 


\subsection{The Analysis by Prakasam}

Prakasam (1996) analyzes several types of English nominal groups containing the item of, which he considers as the construction of "ngp of ngp". In these constructions, the item of is grammatically termed as a pendent. When it comes to the functions of this item, it is treated as a Linker, a Specifier, a Quantificative, a Qualificative, a Possessive or a Process. Here we shall look at the depiction of the nominal group containing a number of. The following two examples in (8) and (9) are taken from Prakasam (1996, p. 575) in which the nominal groups with a number of and the number of occur in the clauses.

(8) He inspected a number of schools, and found their functioning satisfactory.

(9) The number of schools he inspected is much below the target.

The italicized parts are the two nominal groups we are discussing, one functioning as the Complement and the other as the Subject in the clause. In terms of reference, the item their in (8 anaphorically refers to schools in the clause, and therefore the Head of the nominal group falls on schools. In (9), the clause he inspected functions as the Qualifier of the Head schools. The Head is not the Head of the matrix nominal group but the Head of the nominal group following the item of. From the perspective of Subject-Finite agreement, the Head of the matrix nominal group is number, instead. Thus, Prakasam presents two different ways of analyzing the two seemingly similar nominal groups, as shown in Figures 4 and 5.

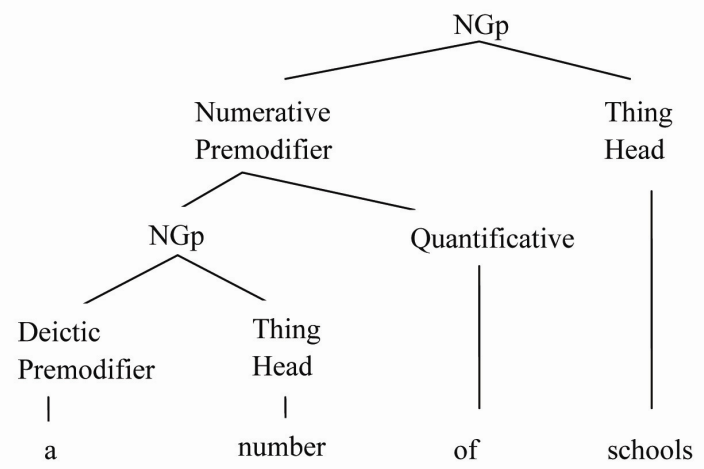

Figure 4. Analysis of the nominal group with a number of by Prakasam (1996, p. 574)

This analysis considers the whole construction a number of as the Numerative, which seems persuasive as $a$ number of has the same function as many. Whatever label it is given, the item of serves as a member in the construction a number of rather than in the structure of the matrix nominal group.

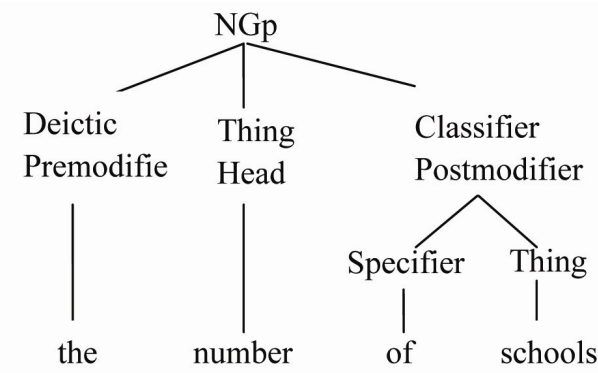

Figure 5. Analysis of the nominal group with the number of by Prakasam (1996, p. 575)

Notice that the structure of the number of is different from that of a number of. Above all, the number of as a whole construction cannot function as the Numerative the same way a number of or any other numerical expressions can. No matter of schools is considered as the Qualifier or the Classifier, it is the Postmodifier in the nominal group modifying the Head. Of in these two cases are respectively termed Quantificative and Specifier by Prakasam (1996) in the sense that the former is associated with the quantifying expression and the latter with specification. 


\section{Discussion}

We have seen the various approaches to the English nominal group containing the numerical expression $a$ number of. In this section we shall discuss the functional structure of the nominal group presented in (1). Fundamentally, this issue will be examined from three perspectives: the functional role of of in the structure, the Head identification of the matrix nominal group, and an analysis by delicacy of the nominal group of this kind.

\subsection{The Functional Role of "of" in the Structure}

On the surface, the two nominal groups five of those books and a large number of those books have an identical structure in that the element after of is $\mathrm{j}$ the same and the element before of has the sense of expressing numbers, exact or inexact. When we explore further, we find that the two structures are completely different.

Fawcett $(2008$, p. 72) points out that the main concern for syntax analysis is to find out "which words go with which to form a unit WITHIN a clause". In other words, the function of a certain item is essentially determined by its location in the unit. We have observed that when it serves as a common preposition, of is embedded in the prepositional phrase, as in the confidant of the three prime ministers, and that when it functions as a selector, of is embedded in the nominal group, as in five of those books. Based on this principle, with regard to the functional role of of in the nominal group in (1), we find that it is embedded neither in the prepositional phrase nor in the nominal group but in the expression a number of. Of is the immediate constituent of a number of, rather than of of those books or a large number of those books.

If we consider the dependency of the element on the structure, we can perceive the distinctive roles of of in three situations. First, the selector is a sister element to other elements, and surely it is an independent element in the nominal group. For instance, of in five of those books does not depend on any element within the nominal group, but on the whole construction of the nominal group. Second, of as a preposition is dependent upon the construction of the prepositional phrase as in the nominal group in (2). In contrast, of in a number of is dependent on the structure of a number of, rather than on that of a number of those books or that of of those books. Only when of occurs together with a number can a number of form a numerical unit in the group having the similar meaning as many and expressing the quantity. In other words, a number of is a whole construction itself and the three elements cannot be separated from each other in order to express a quantifying concept.

Furthermore, the nominal group a large number of those books does not have two referents. On the contrary, it has only one referent that refers to certain books in the real world. A large number of is used to provide more information, the degree of quantity. Thus, selection does not find its place here. On the other hand, of is not a preposition either, for when it is a preposition, the prepositional phrase in which of exists serves as the Qualifier. Obviously enough, this does not comport with this example.

Consequently, a large number of is better to be treated as a quantifying determiner, for it has the same function as any other quantifying determiners do. The above statement suggests that of in the nominal group in (1) is neither a common preposition nor a selector. The function needs to be reconsidered.

\subsection{Head Identification}

We have now concluded that the structure of a large number of those books is distinguished from that of either five of those books or the confidant of three prime ministers. Thus, the Head identification of this nominal group must differ from that of those two nominal groups.

The following three groups of examples are taken from the literature on English grammar (e.g., Alexander, 1991; Zhang, 1995; Zhang, 2002, 2008), and as these are very common and familiar clauses, we will not cite the text and page number from which they are taken. The italicized part is the nominal group that contains the numerical expressions, which have structure identical to that of a number of. Thus, it is proper to treat a number of as a representative example.

\section{Group 1:}

A number of students are playing football on the playground.

Quite a number of very valuable jewels were stolen.

Numbers of people came from all parts of the country to see the exhibition.

\section{Group 2:}

I spent a great deal of my time on this work.

She spends a good deal of money on clothes every year. 
He has spent a large amount /a large sum of money on his new house.

\section{Group 3:}

There are plenty of eggs in the fridge.

There's a lot of / lots of rice in the bag.

A lot of students missed my lecture yesterday.

A lot of the students who missed my lecture yesterday want to borrow my notes.

The difference among the nominal groups italicized in the three groups above resides in the noun following the numerical expressions. The numerical expression is followed by a countable plural noun in Group 1 , by an uncountable noun in Group 2, and by either an uncountable noun or a plural countable noun in Group 3. In all these cases, a number of, a great deal of, a lot of all express the quantity as a whole, and the elements in them cannot be separated from one another to play this role. Evidently, it is not appropriate to treat number, deal, lot as the Head, and the $o f$-phrase after them as the Postmodifier.

For the sake of convenience, we use the example in (1) to discuss. In terms of reference, this nominal group has only one referent, which is books with specific characteristics, a large number of expressing the number and those indicating the deictic property. In addition, in the light of the criterion in the Cardiff Grammar, books is the element expressing the cultural classification, and naturally it is the Head of the matrix nominal group. Moreover, since there is only one referent and of is not embedded in the nominal group, this nominal group does not contain selection of any kind. According to the SFL principle of identifying the Thing of the nominal group, the semantic emphasis falls on books. Consequently, the Thing and the Head coincide with each other in a large number of books.

\subsection{Analysis by Delicacy}

It was argued in the above section that of in the nominal group in (1) is neither a preposition nor a selector. But, questions will be asked: What functional role does the item of play in this construction? How can we do the further analysis of a large number of? This section attempts to answer these questions.

First, what is the word class of of in this case? As Richards, Platt and Platt (2000, p. 333) point out, a particle is "a term sometimes used for a word which cannot readily be identified with any of the main PARTS OF SPEECH (i.e. as a noun, verb, adverb, etc.)". According to this definition, it seems that we can consider of in this linguistic context as a particle. It has no specific meaning, but it cannot be omitted easily, like to in the infinitive expression. Part of speech in the above definition has the same sense as word class in SFL.

On the other hand, however, when we say of is a preposition, we refer to its word class and view it as an element of a prepositional phrase, but when we say it is a selector, we refer to its function in a nominal group. Similarly, even if we can call of a particle in the case of a number of, it is the word class. What we are exploring is its function in this expression.

Halliday (2000) regards three hundred as a word complex, rather than as a nominal group. Can we take a number of as a word complex? We can also say a great number of, a large number of, a small number of, which means we can add modifiers to this expression. As was shown in the example in Group 1, not only number but also a number of can be modified. The fact that quite is used to modify a number of as a whole may suggest that $a$ number of is a structure that can be further analyzed, in terms of delicacy. It is not a word complex.

Actually, a large number of functions as the quantifying determiner and the group itself can be regarded as a quantity group. Fawcett (2000, p. 207) assumes that quantity group "takes its name from the semantic unit of 'quantity' that it realizes, and the 'quantity' that it refers to may be a quantity of a 'thing', a 'situation', a 'quality' or, perhaps surprisingly, a 'quantity'.....”. Based on this definition, we can say a large number of is a quantity group. This group can be analyzed within the framework of the Cardiff Grammar as: $\boldsymbol{a}$ functions as the quantity group determiner (qtd), large as the adjustor (ad), number as the amount (am), and of as the quantity group finisher (qtf). We can borrow the term finisher proffered by Fawcett, although with a slightly different sense.

In addition, the determiner $a$ here has a little difference from the other deictic determiners. When it occurs in expressions like a number of, a lot of, a great deal of, its function is distinguished from its role in expressions like $a$ book. The $a$ in $a$ book can be substituted by other determinative items such as the without the total change of meaning, and the slight difference is only a matter of specification or non-specification. We can also say this book, that book, his book, and so on. But, if the $a$ in a number of is replaced by the or any other determinative items, the function of the whole expression will be entirely changed. In this sense, $a$ in $a$ number of is a special 
determiner, and thus we term it as the quantity group determiner.

In the light of these discussions, we can get the functional structure of the nominal group in (1), as is demonstrated in Figure 6. In the analysis, this nominal group is composed of three elements, the quantifying determiner, the deictic determiner, and the Head. The quantifying determiner is filled by a quantity group that is further composed of four elements. Every item till now has a specific function in the whole nominal group.

So far, the word of in the English nominal group has three labels: preposition, selector, and quantity group finisher. The different labels can be fitted into different circumstances. In other words, different dependency relationship among the elements in the nominal group determines the function of the item of. The numerical expressions such as the ones presented in the three groups in the above section can be analyzed in the same way.

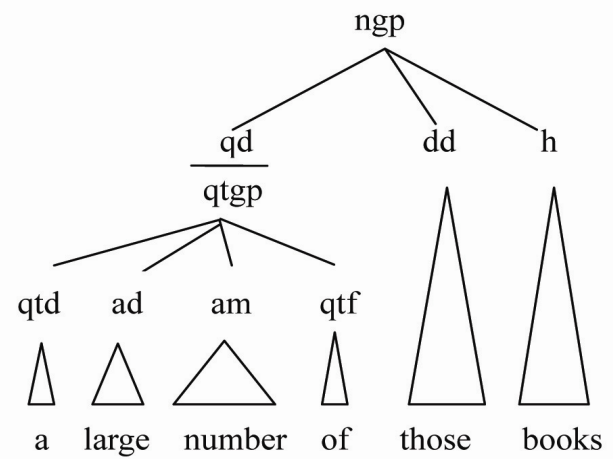

Figure 6. The modified analysis of the nominal group with a number of

\section{Summary and Conclusion}

This paper focuses on the delicate analysis of the English nominal group containing the numerical expression $a$ number of. The point of departure is the comparison of the formal approach and the systemic functional approach on this issue. The formal analysis regards number as the Head, and of-phrase as the Postmodifier of the nominal group. In the systemic functional approach, there are several ways of analyzing the nominal group of this kind. Halliday (2000) treats number as the Head of the nominal group in the logical analysis, while in the experiential analysis a number of together is seen as the Numerative. Prakasam (1996) has the same view, and he also considers of as the Quantificative. The Cardiff Grammar claims that the nominal group a number of those books has the same structure as the nominal group five of those books, and the item of in both cases functions as the selector.

Based on these arguments, we have analyzed the structure of this kind by using the nominal group a large number of those books as an example. The analysis can be summarized as follows: (1) The numerical expression a number of is an independent construction functioning as the Numerative in the nominal group, and every item as an organic element is embedded in the whole construction. In other words, each item is the immediate element of this numerical expression rather than that of the nominal group. (2) The item of in this case is not a common preposition, and it is not appropriate to regard of those books as the prepositional group or the prepositional phrase. In this sense, a number of and the number of are two different structures, and the of in the number of those books is a common preposition having the same function and properties as any other preposition. (3) The nominal group a number of those books does not have the same structure as the group five of those books, either. The item of in five of those books functions as the selector, but it does not in the group a number of those books. The reason is that in the former case of is embedded in the construction as an immediate element of the nominal group but in the latter case it is an immediate element embedded in the numerical expression. We use the term in the Cardiff Grammar and consider it as a quantity group finisher. (4) The item $a$ in the numerical expression is a special determiner, and it is different from the other deictic determiners in that it cannot be substituted by the other determinative items such as the, this, that. It is specifically used in this kind of expression, and we can term it as the quantity group determiner. (5) Head and Thing just coincide with each other in the nominal group $a$ number of those books, and books functions as the Head as well as the Thing.

\section{Acknowledgments}

This research is financially supported by the national project: A Contrastive Study of the English and Chinese 
Nominal Groups: A Systemic Functional Approach. First and foremost, I would like to express my sincere gratitude to my MA and PhD supervisor, Professor Huang Guowen at Sun Yat-sen University, to whom this research owes much. I am also grateful to Professor Robin Fawcett at Cardiff University for his constructive suggestions.

\section{References}

Alexander, L. G. (1991). Longman English Grammar (H. Lei et al., Trans.). Beijing: Foreign Language Teaching and Research Press.

Bloor, T., \& Bloor M. (2001). The Functional Analysis of English: A Hallidayan Approach. Beijing: Foreign Language Teaching and Research Press.

Fawcett, R. P. (2000). A Theory of Syntax for Systemic Functional Linguistics. Amsterdam: Benjamins. http://dx.doi.org/10.1075/cilt.206

Fawcett, R. P. (2006). Establishing the Grammar of "Typicity” in English: An Exercise in Scientific Inquiry. In G. W. Huang, C. G. Chang, \& F. Dai (Eds.), Functional Linguistics as Appliable Linguistics (pp. 159-262). Guangzhou: Sun Yat-sen University Press.

Fawcett, R. P. (2007). Modelling 'Selection' Between Referents in the English Nominal Group. In C. S. Butler, R. H. Downing, \& J. Lavid (Eds.), Functional Perspectives on Grammar and Discourse: in honor of Angela Downing (pp. 165-204). Amsterdam: Benjamins. http://dx.doi.org/10.1075/slcs.85.10faw

Fawcett, R. P. (2008). Invitation to Systemic Functional Linguistics through the Cardiff Grammar: An extension and simplification of Halliday's Systemic Functional Grammar (3rd ed.). London: Equinox.

Halliday, M. A. K. (2000). An Introduction to Functional Grammar (2nd ed.). Beijing: Foreign Language Teaching and Research Press.

Huddleston, R. (1984). Introduction to the Grammar of English. Cambridge: Cambridge University Press. http://dx.doi.org/10.1017/CBO9781139165785

Huddleston, R., \& Pullum, G. K. (2002). The Cambridge Grammar of the English Language. Cambridge: Cambridge University Press.

Prakasam, V. (1996). "NGP of NGP" Constructions: A Functional-Structural Study. In M. Berry, C. S. Butler, R. P. Fawcett, \& G. W. Huang (Eds.), Meaning and Form: Systemic Functional Interpretation. Meaning and Choice in Language: Studies for Michael Halliday (pp. 567-583). Norwood, New Jersey: Ablex.

Quirk, R., Greenbaum, S., Leech, G., \& Svartvik, J. (1985). A Comprehensive Grammar of the English Language. London: Longman.

Richards, J. C., Platt, J., \& Platt, H. (2000). Longman Dictionary of Language Teaching and Applied Linguistics. Beijing: Foreign Language Teaching and Research Press.

Thompson, G. (2000). Introducing Functional Grammar (1st ed.). Beijing: Foreign Language Teaching and Research Press.

Zhang, D. Z. (2002). Zhang Daozhen Yingyu Yufa ("Zhang Daozhen English Grammar”). Beijing: The Commercial Press.

Zhang, D. Z. (2008). Yingyu Yufa Daquan (“A Comprehensive English Grammar”). Beijing: Capital Normal University Press.

Zhang, Z. B. (1995). Xinbian Yingyu Yufa Jiaocheng (“A New English Grammar Course Book”). Shanghai: Shanghai Foreign Language Education Press.

\section{Note}

Note 1. See Alexander, 1991; Zhang, 2002, 2008

\section{Copyrights}

Copyright for this article is retained by the author(s), with first publication rights granted to the journal.

This is an open-access article distributed under the terms and conditions of the Creative Commons Attribution license (http://creativecommons.org/licenses/by/3.0/). 